Research Article

\title{
Integrated Design of Autonomous Orbit Determination and Orbit Control for GEO Satellite Based on Neural Network
}

\author{
Youtao Gao $\mathbb{D},{ }^{1}$ Zhicheng You, ${ }^{1}$ and Bo Xu $\mathbb{D}^{2}$ \\ ${ }^{1}$ School of Astronautics, Nanjing University of Aeronautics and Astronautics, Nanjing 210016, China \\ ${ }^{2}$ School of Aeronautics and Astronautics, Sun Yat-Sen University, Guangzhou 510275, China \\ Correspondence should be addressed to Youtao Gao; ytgao@nuaa.edu.cn
}

Received 6 July 2019; Revised 18 November 2019; Accepted 20 December 2019; Published 21 January 2020

Academic Editor: Paolo Tortora

Copyright (C) 2020 Youtao Gao et al. This is an open access article distributed under the Creative Commons Attribution License, which permits unrestricted use, distribution, and reproduction in any medium, provided the original work is properly cited.

\begin{abstract}
In order to improve the autonomy of a maneuvered GEO satellite which is a member of a navigation satellite system, an integrated design method of autonomous orbit determination and autonomous control was proposed. A neural network state observer was designed to estimate the state of the GEO satellite, with only the intersatellite ranging information as observations. The controller is determined autonomously by another neural network based on the estimated state and the preset correction trajectory. A gradient descent learning method with a forgetting factor was used to derive the weight updating strategy which can satisfy the system's stability and real-time performance. A Lyapunov method was used to prove the stability of both the observer and the controller. The neural network observer can reduce the influence of control on autonomous orbit determination. The neural network controller can improve the robustness of the maneuvered GEO satellite. The simulation results show the effectiveness of this method.
\end{abstract}

\section{Introduction}

Autonomous orbit determination (AOD) of a navigation satellite system can effectively enhance the operational safety of the navigation system, which has attracted the attention of many scholars. The rotation and translation of the entire Earth navigation constellation relative to the inertial reference frame can bring large error to AOD using only interrange measurement. In order to improve the AOD precision, a Lagrangian navigation constellation is added to construct a joint navigation satellite system (JNSS), which can also provide navigation information for interplanetary probe $[1]$.

A special concern of this paper will focus on AOD of a maneuvered GEO satellite which is a member of the JNSS. The GEO satellite plays an important role in the JNSS because of its special position [2]. However, the orbit drift of GEO satellites is quite obvious, so orbit control needs to be carried out about every half month to correct it to the nominal position [3]. The traditional satellite orbit determination method is greatly affected by control. In the process of satellite maneuvering, the modeling error of the traditional thruster is large, which is several orders higher than the perturbation force. Moreover, the pulse control makes the system state discontinuous. Those bring great challenges for precise orbit determination of a maneuvered GEO. Therefore, a maneuvering GEO satellite usually does not provide navigation service until maneuvering is complete. After that, it will take a day or two to resume its navigation function. Generally speaking, maneuver of the GEO satellite greatly reduced its navigation ability. Aiming at this issue, we proposed an integrated design method of autonomous orbit determination and autonomous control during the GEO satellite maneuver.

Some of the works that involved orbit determination of a maneuvered GEO satellite attempted to estimate the thrust acceleration. Song et al. used a first order Markov stochastic process to construct the thrust acceleration model of a maneuvered GEO satellite [4]. Cao et al. used equivalent acceleration to model GEO satellite's thrust acceleration. They established a continuous increasing acceleration by constructing a constant force which is equivalent to the pulse 


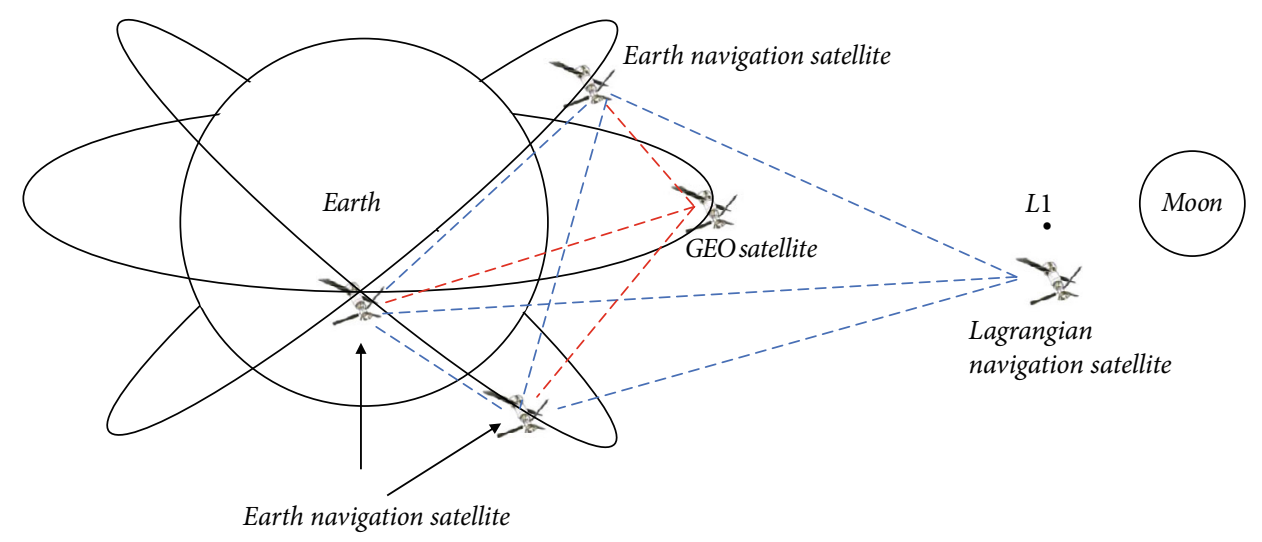

FIGURE 1: GEO satellite observation diagram.

force. The maneuver acceleration was added to the dynamic model as additional disturbance acceleration. Xu et al. proposed a robustly adaptive Kalman filter to estimate the orbit of a maneuvered GEO satellite [5]. Robust estimate is used to resist the influence of measurement outliers. An adaptive factor is added to control the effect of dynamic model errors.

An intelligence observer can be used to estimate states and system parameters without an accurate system model. Furthermore, the neural network has an excellent ability of self-learning and self-adaption [6]. Therefore, it can be used in the AOD of a maneuvered GEO. There are two ways to design an intelligence observer. One is to use a neural network or fuzzifier as the nonlinear observer model; the other is to combine an intelligence algorithm, such as the genetic algorithm, with the conventional orbit determination method to optimize the observer parameters. Vargas and Hemerly presented a neural network-based self-adaptive observer for a multi-input and multioutput system [7]. They used two linear parameterized neural networks to capture the unknown dynamics of the system. Harl et al. used neural network technology to design a reduced-order modified state observer to estimate the states of a satellite [8]. The neural network is used to estimate the unknown perturbation assuming that position can be measured. Gao et al. improve the observer to estimate the state of the navigation satellite with only intersatellite ranging [9]. However, the AOD and control of a maneuvered satellite is not considered.

This paper uses a continuous low thrust control method to control the satellite to the nominal position. The low thrusters have been successfully applied in reality such as pulsed plasma thrusters (PPT). Their working characteristics are as follows: The thrust force is small; control (digital and autonomous control) is convenient and flexible. It can provide a single thrust pulse and can provide equivalent steady-state thrust, which can achieve high-precision control. Compared with traditional chemical thruster impulse control, low thrust control makes the satellite state continuous so as to analyze the stability of the system using the Lyapunov method. The neural network state observer is used to estimate the state of the system. The observation residual will be applied to the weight update law of the neural network, and finally, the observer can be well fitted to the perturbation term which is difficult to model (naturally, it also includes the execution error of the controller). So, this observer will help to recede the effects of model distortion. Furthermore, the neural network controller can maintain strong robustness.

\section{Program Overview of GEO Satellite Autonomous Operation}

The JNSS constructed by a near-Earth constellation and a Lagrangian navigation constellation has been studied in detail in Reference [1], and it points that joint autonomous navigation can suppress the rotational drift of the near-Earth constellation. This paper considers AOD of a GEO satellite during the process of maneuvering. Here, the GEO satellite is a member of the JNSS, and it is controlled frequently. The dynamic equation changes significantly when maneuvering is considered. Whether there are still similar conclusions as Ref. [9] needs further research. For the AOD of the GEO satellites, only the intersatellite range between the GEO satellite and the Earth navigation satellites is used as observation, as shown in Figure 1. The other Earth navigation satellite which is not controlled frequently uses the interrange between the Earth navigation satellite and the Lagrangian satellite to determinate their orbits. For the acquisition of intersatellite ranging, radio ranging is a good choice.

Since the observation frequency of the GEO satellite is higher than the joint autonomous navigation frequency, between the two updates of the linked navigation, the realtime estimated position of the near-Earth satellites observed by the GEO satellite can be directly solved by the satellite precision dynamic equation. Since the interval time is very short ( $15 \mathrm{~min})$, the estimated position calculated by the dynamic equation still has high accuracy. At the same time, the GEO satellite uses the observed data to determine its own state information and provides its own estimated state to the controller to control itself to the target position.

The observer outputs an estimate of the state, and the controller controls the GEO satellite to move along the nominal controlled trajectory and eventually reach the target point. The so-called nominal controlled trajectory is the trajectory $X^{*}$ which was directly calculated by the initial states and the satellite dynamic equation as well as a selected 


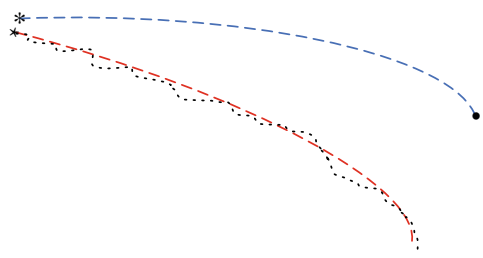

$$
\begin{aligned}
& * \text { : real initial state } \\
& \text { : estimation of initial state } \\
\bullet & : \text { nominal state } \\
--- & : \text { nominal controlled trajectory } \\
--- & : \text { real controlled trajectory } \\
\ldots \ldots & : \text { estimated trajectory }
\end{aligned}
$$

FIgURE 2: The nominal and real controlled trajectories by $\bar{U}$.

control law $\bar{U}$ under the condition of ignoring the influence of error. The sliding mode control is selected in this paper, and the control law $\bar{U}$ is selected as follows [10]:

$$
\left\{\begin{array}{l}
s=c e+\dot{e}=\left[s_{1}, s_{2}, s_{3}\right]^{T}, \\
\bar{U}=-U_{\max } \operatorname{sign}(s),
\end{array}\right.
$$

where $e$ is the difference between the current real states and the nominal states of the satellite, $U_{\max }$ is the maximum acceleration that ion thruster can provide, and $c$ is the position error scale coefficient, which is a $3 \times 3$ positive definite diagonal matrix.

Due to the influence of ignored error on the real system, the real controlled trajectory of the system controlled by $\bar{U}$ will gradually deviate from $X^{*}(t)$, as shown in Figure 2 . The control output by which the real states of the satellite is controlled near $X^{*}(t)$ and finally reaches the nominal state is called the nominal control quantity $U^{*}$. $U^{*}$ is difficult to be calculated directly, but it can be fitted through the neural network by the difference between the current estimated state and $X^{*}(t)$ and the difference between the actual observation and the theoretical observation calculated by $X^{*}(t)$.

\section{System Equations for a Maneuvered Satellite}

The dynamic equation of the maneuvered GEO satellite is expressed as

$$
\ddot{r}=-\frac{\mu}{r^{3}} r+a_{f}+a_{U},
$$

where $\mu$ is the gravitational constant of the celestial body, $a_{f}$ is the sum of perturbation accelerations, and $a_{U}$ is the maneuvering acceleration. For a GEO satellite, the drift position is usually not very large, and the nominal orbit of the GEO satellite is a circular orbit. Therefore, we can use the relative motion equation to describe the orbit variation. As shown in Figure 3, $C$ is the real position of the GEO satellite and $S$ is the target position. A local vertical local horizontal coordinate $S$ - $x y z$ is attached to $S$ with $x$ axis in the radial direction of $S, z$ axis is parallel to the Earth's rotation angular momentum, and $y$ axis is established by the right-hand rule.

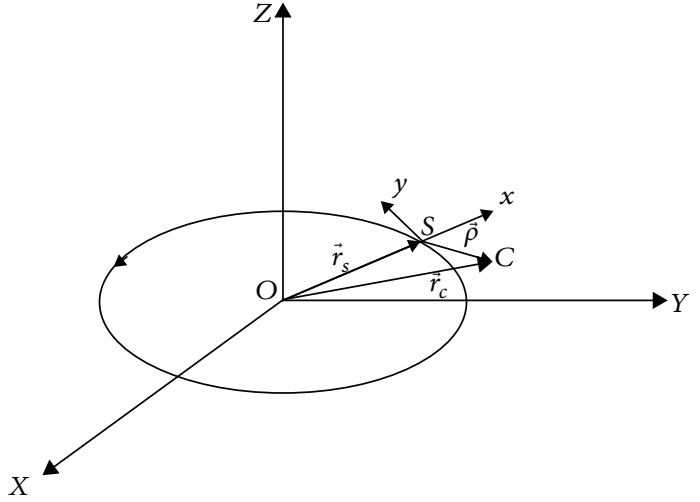

FIgURE 3: The relative motion coordinate system and the Earth's inertial coordinate system.

Based on existing research, the relative motion equation can be written as $[11,12]$

$$
\left\{\begin{array}{l}
\ddot{x}-2 n \dot{y}-3 n^{2} x=f_{x}+u_{x}, \\
\ddot{y}+2 n \dot{x}=f_{y}+u_{y}, \\
\ddot{z}+n^{2} z=f_{z}+u_{z},
\end{array}\right.
$$

where $f_{x}, f_{y}$, and $f_{z}$ are the projections of $a_{f}$ in the three axes of the $S$ - $x y z$ coordinate system and $u_{x}, u_{y}$, and $u_{z}$ are the projections of $a_{U}$.

We define $X=\left[x, y, z, v_{x}, v_{y}, v_{z}\right]^{T}, f=\left[\begin{array}{lll}f_{x} & f_{y} & f_{z}\end{array}\right]^{T}$, and $U=\left[\begin{array}{lll}u_{x} & u_{y} & u_{z}\end{array}\right]^{T}$, then Equation (3) can be expressed as

$$
\dot{X}=A \cdot X+B(f+U)
$$

where

$$
A=\left[\begin{array}{cccccc}
0 & 0 & 0 & 1 & 0 & 0 \\
0 & 0 & 0 & 0 & 1 & 0 \\
0 & 0 & 0 & 0 & 0 & 1 \\
3 n^{2} & 0 & 0 & 0 & 2 n & 0 \\
0 & 0 & 0 & -2 n & 0 & 0 \\
0 & 0 & -n^{2} & 0 & 0 & 0
\end{array}\right],
$$

$$
B=\left[\begin{array}{lll}
0 & 0 & 0 \\
0 & 0 & 0 \\
0 & 0 & 0 \\
1 & 0 & 0 \\
0 & 1 & 0 \\
0 & 0 & 1
\end{array}\right],
$$

and $n$ is the magnitude of $S$ angular velocity. 


\section{Design of Observer and Controller Based on Neural Networks}

As we defined earlier, $X^{*}(t)$ is the nominal controlled trajectory of the GEO satellite, and in the relative motion coordinate system, it satisfies

$$
\dot{X}^{*}=A \cdot X^{*}+B\left(f+U^{*}\right) \text {. }
$$

The controller's task is to control the satellite to reach the target point along the trajectory of Equation (6). $X^{*}$ can be easily obtained by early calculation, but the states of the satellite $X$ cannot be directly observed; we can directly obtain only the intersatellite ranging information $Y$, so it is necessary to establish a state observer based on the actual observation. We defined $\widehat{X}$ as the estimated state of a maneuvered GEO satellite, and a more common form of the Lemberger state observer is shown in Equation (7) [8]:

$$
\dot{\hat{X}}=A \cdot \widehat{X}+B(\widehat{U}+\widehat{f})+K\left(Y_{t}-\widehat{Y}_{t}\right), \quad \widehat{Y}_{t}=C \cdot \widehat{X},
$$

where $\widehat{f}$ is an estimated value of $f, \widehat{U}$ is an estimated value of $U^{*}, K$ is a feedback gain matrix, and $A-K C$ is a Hurwitz matrix. $Y_{t}=C X$.

Our true observations are intersatellite ranging which can be expressed as $[13,14]$

$$
Y=\rho(X, t)=\sqrt{\left(x-x_{i}\right)^{2}+\left(y-y_{i}\right)^{2}+\left(z-z_{i}\right)^{2}},
$$

where $[x, y, z]$ and $\left[x_{i}, y_{i}, z_{i}\right]$ are the position variable of the GEO satellite and satellite $i$ in a JNSS [8]. Expanding Equation (8) in a Taylor series with respect to estimated state $\widehat{X}$ and neglecting high-order components, we get the linearized relation between $\tilde{Y}=(Y-\widehat{Y})$ and $E_{o}=(X-\widehat{X})$ as

$$
\tilde{Y}_{o}=C_{o} \cdot E_{o}, \quad C_{o}=\left.\frac{\partial Y}{\partial X^{T}}\right|_{X=\hat{X}} .
$$

Due to the universal function approximation characteristics of neural networks [7], $U^{*}$ and $f$ can be expressed in the form of Equation (10), where $\varepsilon_{c}\left(X^{*}\right)$ and $\varepsilon_{o}(X)$ represent the fitting errors, $\varepsilon_{\max }^{c}$ and $\varepsilon_{\max }^{o}$ are the upper bounds of the allowed error, $W_{c}$ and $W_{o}$ represent the ideal neural network weights, and $\phi_{c}\left(X^{*}\right)$ and $\phi_{o}(X)$ are bounded basis functions:

$$
\begin{gathered}
U^{*}=W_{c} \cdot \phi_{c}\left(X^{*}\right)+\varepsilon_{c}\left(X^{*}\right), \quad\left\|\varepsilon_{c}\left(X^{*}\right)\right\| \leq \varepsilon_{\max }^{c}, \\
f=W_{o} \cdot \phi_{o}(X)+\varepsilon_{o}(X), \quad\left\|\varepsilon_{o}(X)\right\| \leq \varepsilon_{\max }^{o} .
\end{gathered}
$$

$\widehat{U}$ and $\widehat{f}$ can be expressed in the form of Equation (11), where $\widehat{W}_{c}$ and $\widehat{W}_{o}$ represent estimates of the weights of the two ideal neural networks, respectively:

$$
\begin{aligned}
& \widehat{U}=\widehat{W}_{c} \cdot \phi_{c}(\widehat{X}), \\
& \widehat{f}=\widehat{W}_{o} \cdot \phi_{o}(\widehat{X}) .
\end{aligned}
$$

In order to eliminate the influence of the initial estimation error, $\widehat{U}$ also needs to add an output feedback term in Equation (11); that is, the actual output of the controller is Equation (12), where $H$ is the feedback matrix which makes matrix $(A-B C H)$ a Hurwitz matrix:

$$
\widehat{U}=\widehat{W}_{c} \cdot \phi_{c}(\widehat{X})-H\left(Y-Y^{*}\right) .
$$

The estimation error of the observer neural network weight will increase the observation error $\tilde{Y}$, and the estimation error of the controller neural network weight will cause $\tilde{Y}_{c}=Y-Y^{*}$ to increase. Design the cost function of the weight update as $J_{o}=(1 / 2) \tilde{Y}_{o}^{T} \tilde{Y}_{o}$ and $J_{c}=(1 / 2) \tilde{Y}_{c}^{T} \tilde{Y}_{c}$.

The neural network weight update law is obtained by using the gradient descent algorithm with correction term $[15,16]$ :

$$
\dot{\hat{W}}=-\eta\left(\frac{\partial J}{\partial \widehat{W}}\right)-\kappa\|\tilde{Y}\| \widehat{W}
$$

where $\eta$ is the learning rate constant and $\kappa$ is the forgetting factor.

According to derivation rule of compound function,

$$
\frac{\partial J_{o}}{\partial \widehat{W}_{o}^{T}}=\frac{\partial J_{o}}{\partial \tilde{Y}_{o}} \cdot \frac{\partial \tilde{Y}_{o}}{\partial \widehat{X}} \cdot \frac{\partial \widehat{X}}{\partial \widehat{f}} \cdot \frac{\partial \widehat{f}}{\partial \widehat{W}_{o}^{T}}=-\tilde{Y}_{o}^{T} \cdot C_{o} \cdot \frac{\partial \widehat{X}}{\partial \widehat{f}} \cdot \phi_{o}(\widehat{X})
$$

We assume $\partial \dot{\hat{X}} / \partial \widehat{f}=A_{c k}(\partial \dot{\hat{X}} / \partial \widehat{f})+B \approx 0$ [17]; then, we can get

$$
\begin{aligned}
\frac{\partial J_{o}}{\partial \widehat{W}_{o}^{T}} & =-\tilde{Y}_{o}^{T} \cdot C_{o} \cdot \frac{\partial \widehat{X}}{\partial \widehat{f}} \cdot \widehat{\phi}_{o} \\
& =-\tilde{Y}_{o}^{T} \cdot C_{o} \cdot\left(-B A_{c k}^{-1}\right) \cdot \widehat{\phi}_{o} \\
& =E_{o}^{T} \cdot C_{o}^{T} C_{o} B A_{c k}^{-1} \cdot \widehat{\phi}_{o},
\end{aligned}
$$

where $\widehat{\phi}_{o}=\phi_{o}(\widehat{X})$.

Substituting Equation (15) into Equation (13),

$$
\dot{\hat{W}}_{o}=-\eta_{o}\left(E_{o}^{T} \cdot C_{o}^{T} C_{o} B A_{c k}^{-1} \cdot \widehat{\phi}_{o}\right)^{T}-\kappa_{o}\left\|C_{o} E_{o}\right\| \widehat{W}_{o} .
$$

It should be noted that since the ideal weight $W_{o}$ is constant, $\dot{\tilde{W}}_{o}=\dot{W}_{o}-\dot{\widehat{W}}_{o}=-\dot{\hat{W}}_{o}$. 


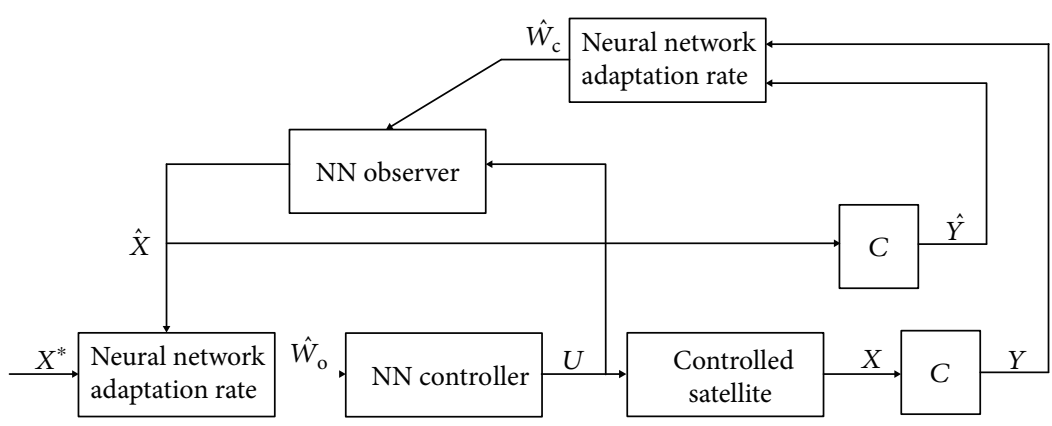

Figure 4: The structure of the system.

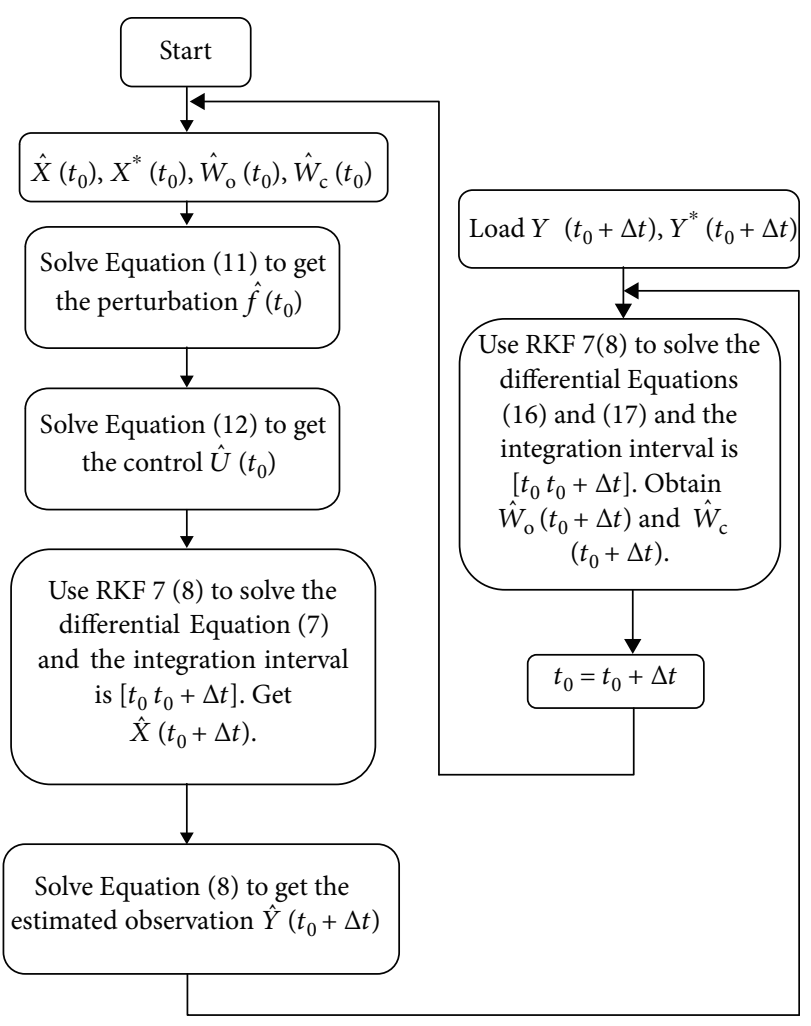

Figure 5: The flow chart of system operation.

The weight update law in the controller can be obtained in the same way:

$$
\dot{\widehat{W}}_{c}=-\eta_{c}\left(E_{c}^{T} \cdot C_{c}^{T} C_{c} B A_{B H}^{-1} \cdot \widehat{\phi}_{c}\right)^{T}-\kappa_{c}\left\|C_{c} E_{c}\right\| \widehat{W}_{c},
$$

where $\widehat{\phi}_{c}=\phi_{c}(\widehat{X})$ and $E_{c}=X-X^{*}$ representative control error.

After establishing two neural networks and giving the weight update law, the system structure diagram can be established as shown in Figure 4, where "NN" is an abbreviation for the neural network.

Suppose the time interval for GEO satellites to obtain intersatellite observations is $\Delta t$. Figure 5 shows the workflow of the observer and controller in the system.

\section{System Stability Proof Based on Lyapunov Method}

Refer to Reference [13], if only intersatellite range is chosen as observation, three intersatellite ranges are required for AOD: $Y=\left[\begin{array}{lll}\rho_{1} & \rho_{2} & \rho_{3}\end{array}\right]^{T}$.

If define $\tilde{\phi}_{c}=\phi_{c}\left(X^{*}\right)-\phi_{c}(\widehat{X}), \tilde{W}_{c}^{T}=W_{c}^{T}-\widehat{W}_{c}^{T}$. Subtraction of Equations (4) and (6) can be obtained:

$$
\begin{aligned}
\dot{E}_{c}= & A \cdot E_{c}+B\left(\widehat{U}-U^{*}\right) \\
= & A \cdot E_{c}+B\left(\widehat{W}_{c}^{T} \cdot \phi_{c}(\widehat{X})-H\left(Y-Y^{*}\right)-W_{c}^{T} \cdot \phi_{c}\left(X^{*}\right)-\varepsilon_{c}\right) \\
= & A \cdot E_{c}-B \cdot H C E_{c}+B \cdot \varepsilon_{c} \\
& +B\left[W_{c}^{T}\left(\phi_{c}\left(X^{*}\right)-\phi_{c}(\widehat{X})\right)+\left(W_{c}^{T}-\widehat{W}_{c}^{T}\right) \phi_{c}(\widehat{X})\right] \\
= & A_{B H} E_{c}+B \tilde{W}_{c}^{T} \widehat{\phi}_{c}+w_{c}(t), \\
\tilde{Y}_{c}= & C_{c} E_{c},
\end{aligned}
$$

where $w_{c}(t)=B \cdot\left(W_{c}^{T} \tilde{\phi}_{c}+\varepsilon_{c}\right) \cdot C_{c}$ is similar to $C_{o}$ in Equation (10) and can be obtained by the same method. Since the compression function is bounded, $\left\|w_{o}(t)\right\|_{F} \leq \bar{w}_{o}$.

The same method as above, subtraction of Equations (4) and (7), and define $\tilde{\phi}_{o}=\phi_{o}(X)-\phi_{o}(\widehat{X}), \tilde{W}_{o}^{T}=W_{o}^{T}-\widehat{W}_{o}^{T}$. We can obtain

$$
\begin{aligned}
\dot{E}_{o} & =A E_{o}+B(f-\widehat{f})-K(Y-\widehat{Y}) \\
& =(A-K C) E_{o}+B\left[W_{o}^{T} \cdot \phi_{o}(X)+\varepsilon_{o}-\widehat{W}_{o}^{T} \cdot \phi_{o}(\widehat{X})+v_{o}\right] \\
& =A_{c k} E_{o}+B \cdot \tilde{W}_{o}^{T} \widehat{\phi}_{o}+w_{o}(t), \\
\tilde{Y}_{o} & =C_{o} E_{o},
\end{aligned}
$$

where $w_{o}(t)=B \cdot\left(W_{o}^{T} \tilde{\phi}_{o}+\varepsilon_{o}\right),\left\|w_{o}(t)\right\|_{F} \leq \bar{w}_{o}$.

We define the Lyapunov function as follows:

$$
V_{o}=\frac{1}{2} E_{o}^{T} P_{o} E_{o}+\frac{1}{2} E_{c}^{T} P_{c} E_{c}+\frac{1}{2} \operatorname{tr}\left[\tilde{W}_{o}^{T} \tilde{W}_{o}\right]+\frac{1}{2} \operatorname{tr}\left[\tilde{W}_{c}^{T} \tilde{W}_{c}\right],
$$


TABLE 1: Initial state of each satellite.

\begin{tabular}{lcccccc}
\hline & $X(\mathrm{~m})$ & $Y(\mathrm{~m})$ & $Z(\mathrm{~m})$ & $V_{X}\left(\mathrm{~ms}^{-1}\right)$ & $V_{Y}\left(\mathrm{~ms}^{-1}\right)$ & $V_{Z}\left(\mathrm{~ms}^{-1}\right)$ \\
\hline GEO & 42240618.92 & -184065.36 & -79979.923 & 13.385657 & 3071.8300 & 0.011524 \\
PRN1 & 9852071.558 & -24629399.99 & 1064965.918 & 2100.5759 & 724.5559 & -3175.7033 \\
PRN8 & -15037328.43 & -11415774.61 & 18486885.74 & 3195.1517 & -1507.9377 & 1636.9601 \\
PRN31 & 15116130.71 & -18972585.47 & 10208754.56 & 1194.7484 & 2468.0586 & 2786.5419 \\
\hline
\end{tabular}

TABLE 2: Comparison of three different trajectories.

\begin{tabular}{|c|c|c|c|}
\hline & Real trajectory & Estimated trajectory & Nominal trajectory \\
\hline Equations to be used & $\begin{array}{l}\text { (1) } \ddot{r}=-\left(\mu / r^{3}\right) r \\
\text { (2) } \ddot{r}=-\left(\mu / r^{3}\right) r+a_{f}+a_{U}\end{array}$ & $\dot{\hat{X}}=A \cdot \widehat{X}+B(\widehat{U}+\widehat{f})+K\left(Y_{t}-\widehat{Y}_{t}\right)$ & $\dot{X}^{*}=A \cdot X^{*}+B \bar{U}$ \\
\hline Solving steps & $\begin{array}{l}\text { Solve Eq }(1) \text {, determine the } \\
\text { motion coordinate } S \text { - } x y z \\
\text { from the result; solve Eq (2), } \\
\text { transform the result into } S \text { - } x y z\end{array}$ & $\begin{array}{l}\text { Solve the equation online } \\
\text { according to the flowchart } \\
\text { shown in Figure } 5\end{array}$ & $\begin{array}{l}\text { Substitute the initial } \\
\text { state values on the } \\
\text { coordinate system } \\
\text { S- } x y z \text { and solve the } \\
\text { equation offline }\end{array}$ \\
\hline
\end{tabular}

where $P_{o}$ and $P_{c}$ are positive definite matrices that satisfy

$$
\begin{array}{ll}
-Q_{o}=P_{o} A_{c k}+A_{c k}^{T} P_{o}, & Q_{o}>0, \\
-Q_{c}=P_{c} A_{B H}+A_{B H}^{T} P_{c}, & Q_{o}>0 .
\end{array}
$$

The time derivative of (20) is given by

$$
\begin{aligned}
\dot{V}_{o}= & E_{o}^{T} P_{o} \dot{E}_{o}+E_{c}^{T} P_{c} \dot{E}_{c}+\operatorname{tr}\left[\tilde{W}_{o}^{T} \dot{\tilde{W}}_{o}\right]+\operatorname{tr}\left[\tilde{W}_{c}^{T} \dot{\tilde{W}}_{c}\right] \\
= & -\frac{1}{2} E_{o}^{T} Q_{o} E_{o}-\frac{1}{2} E_{c}^{T} Q_{c} E_{c}+E_{o}^{T} P_{o} B \cdot \tilde{W}_{o}^{T} \widehat{\phi}_{o} \\
& +E_{o}^{T} P_{o} w_{o}(t)+E_{c}^{T} P_{c} B \cdot \tilde{W}_{c}^{T} \widehat{\phi}_{c}+E_{c}^{T} P_{c} w_{c}(t) \\
& +\operatorname{tr}\left[\eta_{o} \tilde{W}_{o}^{T}\left(E_{o}^{T} \cdot C_{o}^{T} C_{o} B A_{c k}^{-1} \cdot \widehat{\phi}_{o}\right)^{T}\right] \\
& +\operatorname{tr}\left[\eta_{c} \tilde{W}_{c}^{T}\left(E_{c}^{T} \cdot C_{c}^{T} C_{c} B A_{B H}^{-1} \cdot \widehat{\phi}_{c}\right)^{T}\right] \\
& +\operatorname{tr}\left[\kappa_{o}\left\|C_{o} E_{o}\right\| \tilde{W}_{o}^{T} \widehat{W}_{o}\right]+\operatorname{tr}\left[\kappa_{c}\left\|C_{c} E_{c}\right\| \tilde{W}_{c}^{T} \widehat{W}_{c}\right] .
\end{aligned}
$$

The following relations are reliable:

$$
\begin{aligned}
& -\frac{1}{2} E_{o}^{T} Q_{o} E_{o} \leq-\frac{1}{2} \lambda_{\min }\left(Q_{o}\right)\left\|E_{o}\right\|^{2}, \\
& -\frac{1}{2} E_{c}^{T} Q_{c} E_{c} \leq-\frac{1}{2} \lambda_{\min }\left(Q_{c}\right)\left\|E_{c}\right\|^{2}, \\
& \operatorname{tr}\left[\tilde{W}_{o}^{T} \widehat{W}_{o}\right] \leq W_{\max }^{o}\left\|\tilde{W}_{o}\right\|_{F}-\left\|\tilde{W}_{o}\right\|_{F}^{2}, \\
& \operatorname{tr}\left[\tilde{W}_{c}^{T} \widehat{W}_{c}\right] \leq W_{\max }^{c}\left\|\tilde{W}_{c}\right\|_{F}-\left\|\tilde{W}_{c}\right\|_{F}^{2} .
\end{aligned}
$$

Substituting Equation (23) into Equation (22), it yields

$$
\begin{aligned}
\dot{V} \leq & -\frac{1}{2} \lambda_{\max }\left(Q_{o}\right)\left\|E_{0}\right\|^{2}+\left\|E_{0}\right\| \cdot\left[\left\|P_{0}\right\| \cdot\left(\delta\left\|\tilde{W}_{o}\right\|+\bar{w}_{o}\right)\right. \\
& \left.+\left\|\tilde{W}_{o}\right\| \cdot \delta\left\|l_{o}\right\|+\kappa_{o}\left\|C_{o}\right\| \cdot\left(W_{\max }^{o} \cdot\left\|\tilde{W}_{o}\right\|-\left\|\tilde{W}_{o}\right\|^{2}\right)\right] \\
& -\frac{1}{2} \lambda_{\max }\left(Q_{c}\right)\left\|E_{c}\right\|^{2}+\left\|E_{c}\right\| \cdot\left[\left\|P_{c}\right\| \cdot\left(\delta\left\|\tilde{W}_{c}\right\|+\bar{w}_{c}\right)\right. \\
& \left.+\left\|\tilde{W}_{c}\right\| \cdot \delta\left\|l_{c}\right\|+\kappa_{c}\left\|C_{c}\right\| \cdot\left(W_{\max }^{o} \cdot\left\|\tilde{W}_{c}\right\|-\left\|\tilde{W}_{c}\right\|^{2}\right)\right] \\
= & -\frac{1}{2} \lambda_{\max }\left(Q_{o}\right)\left\|E_{0}\right\|^{2}-\frac{1}{2} \lambda_{\max }\left(Q_{c}\right)\left\|E_{c}\right\|^{2}+\left\|E_{0}\right\| \\
& \cdot\left[-\kappa_{o}\left\|C_{o}\right\| \cdot\left\|\tilde{W}_{o}\right\|^{2}+\left(\delta \cdot\left\|P_{0}\right\|+\delta\left\|l_{o}\right\|+\kappa_{o}\left\|C_{o}\right\| W_{\max }^{o}\right)\right. \\
& \left.\cdot\left\|\tilde{W}_{o}\right\|+\left\|P_{0}\right\| \cdot \bar{w}_{o}\right]+\left\|E_{c}\right\| \cdot\left[-\kappa_{c}\left\|C_{c}\right\| \cdot\left\|\tilde{W}_{c}\right\|^{2}\right. \\
& \left.+\left(\delta \cdot\left\|P_{c}\right\|+\delta\left\|l_{c}\right\|+\kappa_{c}\left\|C_{c}\right\| W_{\max }^{c}\right) \cdot\left\|\tilde{W}_{c}\right\|+\left\|P_{c}\right\| \cdot \bar{w}_{c}\right] .
\end{aligned}
$$

$\delta$ is a positive constant, and $\phi_{o}(\cdot) \leq \delta, \phi_{c}(\cdot) \leq \delta . l_{o}=C_{o}^{T}$ $C_{o} B A_{c k}^{-1}, l_{c}=C_{c}^{T} C_{c} B A_{B H}^{-1}$.

Further simplification (24) is available:

$$
\begin{aligned}
\dot{V} \leq & -\frac{1}{2} \lambda_{\max }\left(Q_{o}\right)\left\|E_{0}\right\|^{2}+\left\|E_{0}\right\| \\
& \cdot\left[\frac{\left(\delta \cdot\left\|P_{0}\right\|+\delta\left\|l_{o}\right\|+\kappa_{o}\left\|C_{o}\right\| W_{\max }^{o}\right)^{2}}{4 \kappa_{o}\left\|C_{o}\right\|}+\left\|P_{0}\right\| \cdot \bar{w}_{o}\right] \\
& -\frac{1}{2} \lambda_{\max }\left(Q_{c}\right)\left\|E_{c}\right\|^{2}+\left\|E_{c}\right\| \\
& \cdot\left[\frac{\left(\delta \cdot\left\|P_{c}\right\|+\delta\left\|l_{c}\right\|+\kappa_{c}\left\|C_{c}\right\| W_{\max }^{c}\right)^{2}}{4 \kappa_{c}\left\|C_{c}\right\|}+\left\|P_{c}\right\| \cdot \bar{w}_{c}\right] .
\end{aligned}
$$



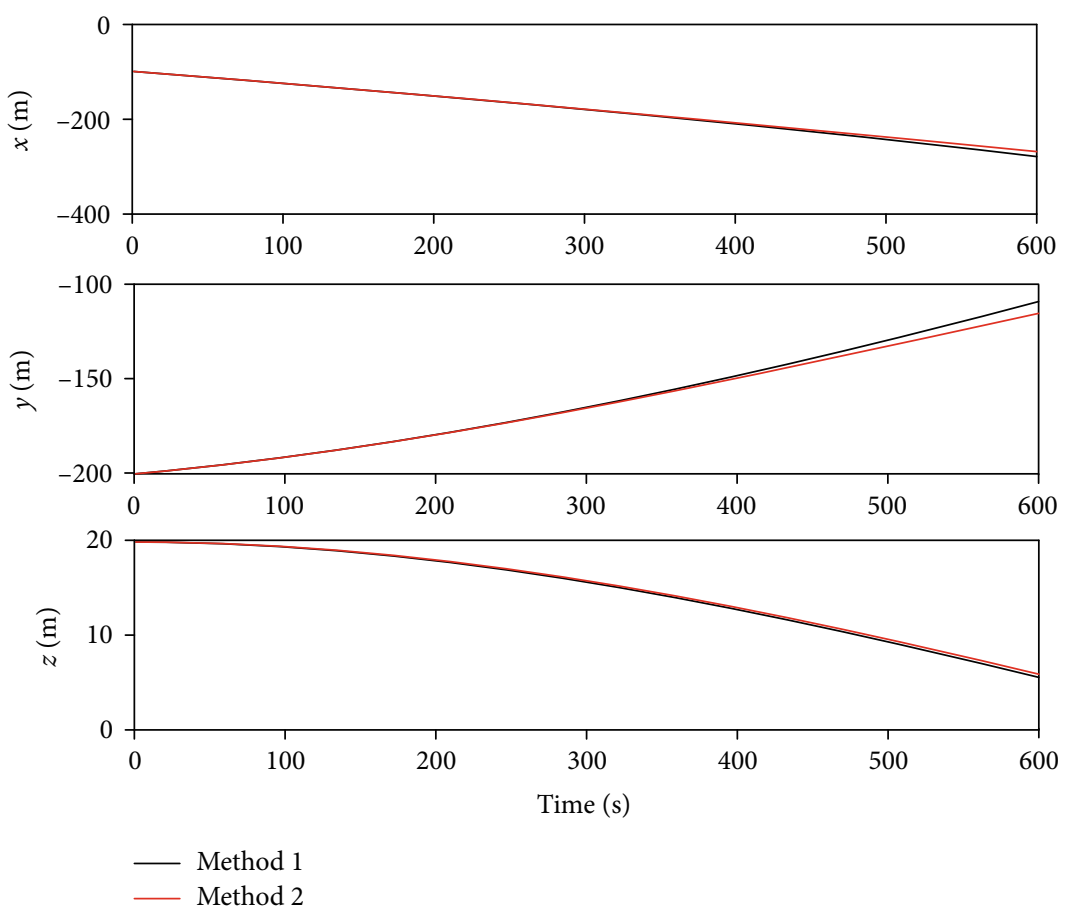

FIGURE 6: Schematic diagram of the position change of GEO satellites near the nominal position without control.

Let $\quad M_{1}=\left(\delta \cdot\left\|P_{0}\right\|+\delta\left\|l_{o}\right\|+\kappa_{o}\left\|C_{o}\right\| W_{\max }^{o}\right)^{2} / 4 \kappa_{o}\left\|C_{o}\right\|, \quad$ Obviously, if $M_{2}=\left(\delta \cdot\left\|P_{c}\right\|+\delta\left\|l_{c}\right\|+\kappa_{c}\left\|C_{c}\right\| W_{\max }^{c}\right)^{2} / 4 \kappa_{c}\left\|C_{c}\right\|$.

$$
\begin{array}{r}
\left\|E_{0}\right\| \geq \frac{-\left(M_{1}+\left\|P_{0}\right\| \cdot \bar{w}_{o}\right)+\sqrt{\left(M_{1}+\left\|P_{0}\right\| \cdot \bar{w}_{o}\right)^{2}+\left(\lambda_{\max }\left(Q_{o}\right)\left(M_{2}+\left\|P_{c}\right\| \cdot \bar{w}_{c}\right)^{2} / \lambda_{\max }\left(Q_{c}\right)\right)}}{\lambda_{\max }\left(Q_{o}\right)}, \\
\text { or }\left\|E_{c}\right\| \geq \frac{-\left(M_{2}+\left\|P_{c}\right\| \cdot \bar{w}_{c}\right)+\sqrt{\left(M_{2}+\left\|P_{c}\right\| \cdot \bar{w}_{c}\right)^{2}+\left(\lambda_{\max }\left(Q_{c}\right)\left(M_{1}+\left\|P_{o}\right\| \cdot \bar{w}_{o}\right)^{2} / \lambda_{\max }\left(Q_{o}\right)\right)}}{\lambda_{\max }\left(Q_{c}\right)},
\end{array}
$$

$\dot{V}$ is negative. So, it can be proved that $E_{o}$ and $E_{c}$ are bounded.

The results of the above derivation show that the control error $E_{c}$ and state estimation error $E_{o}$ of the system are bounded. The system is a stable system. The bounds of $E_{c}$ and $E_{o}$ are related to the parameters we define which can be chosen appropriately to minimize the error.

\section{Simulation and Analysis}

In order to verify the feasibility and specific effects of the method, the following three sets of experiments were designed. Experiment 1 shows the GEO satellite drift when uncontrolled. The second experiment is the traditional UKF filtering method, and the third experiment is the neural network method proposed in this paper. Table 1 gives the initial values of the GEO satellites and the observed GPS satellites. Before the three sets of experiments, the author used STK software to simulate the intersatellite link, and the link between the sat- ellites did not appear to be occluded by the Earth. Therefore, it can be considered that in these experiments, intersatellite ranging can always be achieved. For the controller, to achieve forward and reverse outputs in three directions, the six thrusters should be divided into three groups and mounted on three axes perpendicular to each other of the satellite. In order not to affect the satellite attitude, the thrust direction of each controller must pass through the mass center.

In order to clearly express the simulation ideas, Table 2 compares the generation methods of the real trajectory, estimated trajectory, and nominal trajectory in detail.

In the simulation, controller error and unknown perturbation are uniformly represented by trigonometric functions, because perturbations of satellites often do not meet the Gaussian distribution while their changes are generally continuous or near continuous.

The calculated value of $u$ will be used in the dynamical model of the observer, and the real dynamical model contains 

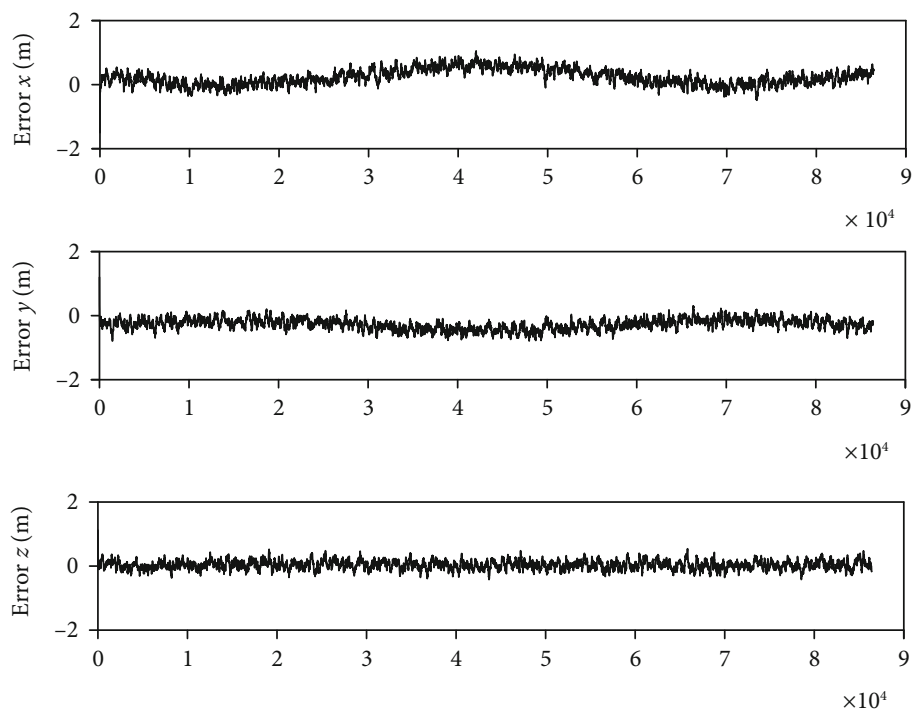

FIgURe 7: Position estimation error (UKF).
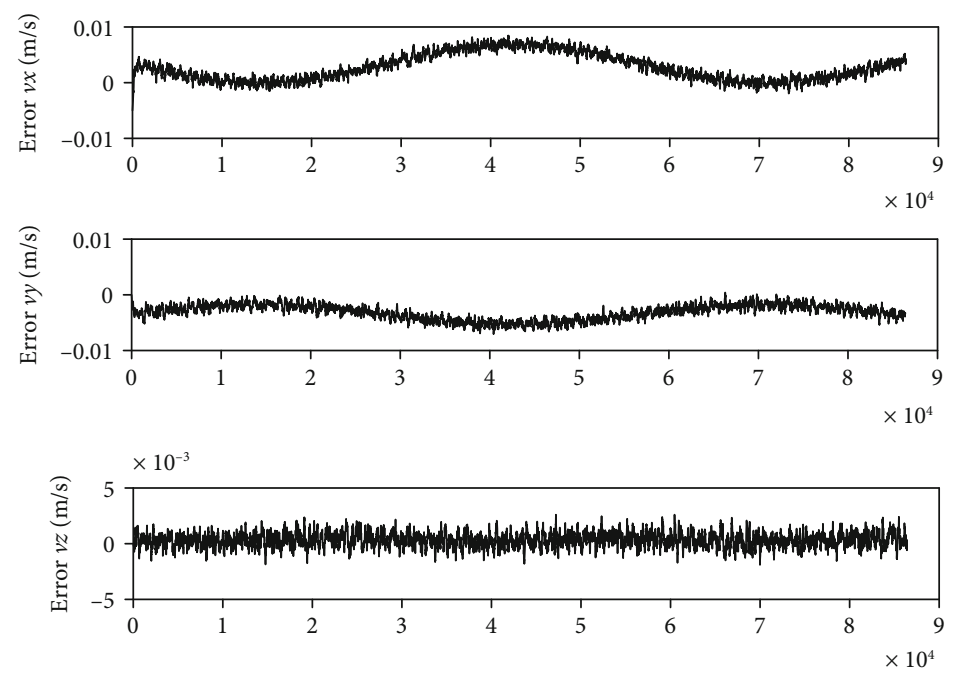

FIgURE 8: Velocity estimation error (UKF).

the controller error and other unknown perturbations. In other words, the calculated control amount can be considered to be known, and the control error and other unknown perturbations are modeling errors of the filter equation.

In the relative motion coordinate system, the GEO satellite initial deviation error is set to $X_{0}=[100 \mathrm{~m} 200 \mathrm{~m}$ $-20 \mathrm{~m} 0.01 \mathrm{~m} / \mathrm{s} \quad 0 \mathrm{~m} / \mathrm{s} \quad 0 \mathrm{~m} / \mathrm{s}$ ].

Experiment 1. The drift experiment of GEO satellite without control. Two methods are used: the first is to integrate the relative motion Equation (3) directly in the relative motion coordinate system, while the second is to solve equation (2) and convert the result to the relative motion coordinate system by means of coordinate transformation. The results obtained are shown in Figure 6. It can be seen from the figure that the GEO satellite will continue to move away from the target position without control, and the relative motion equation can precisely show its deviation from the target point.

Experiment 2. The UKF filter used for orbit determination. In the experiment, obtain an observation every 10 seconds. Calculate the control amount after each step of filtering, given by Equation (1). The initial estimated value of the filter is

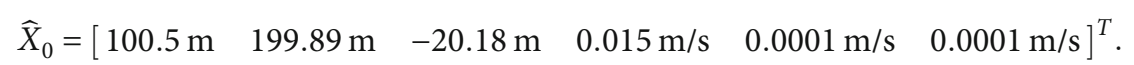



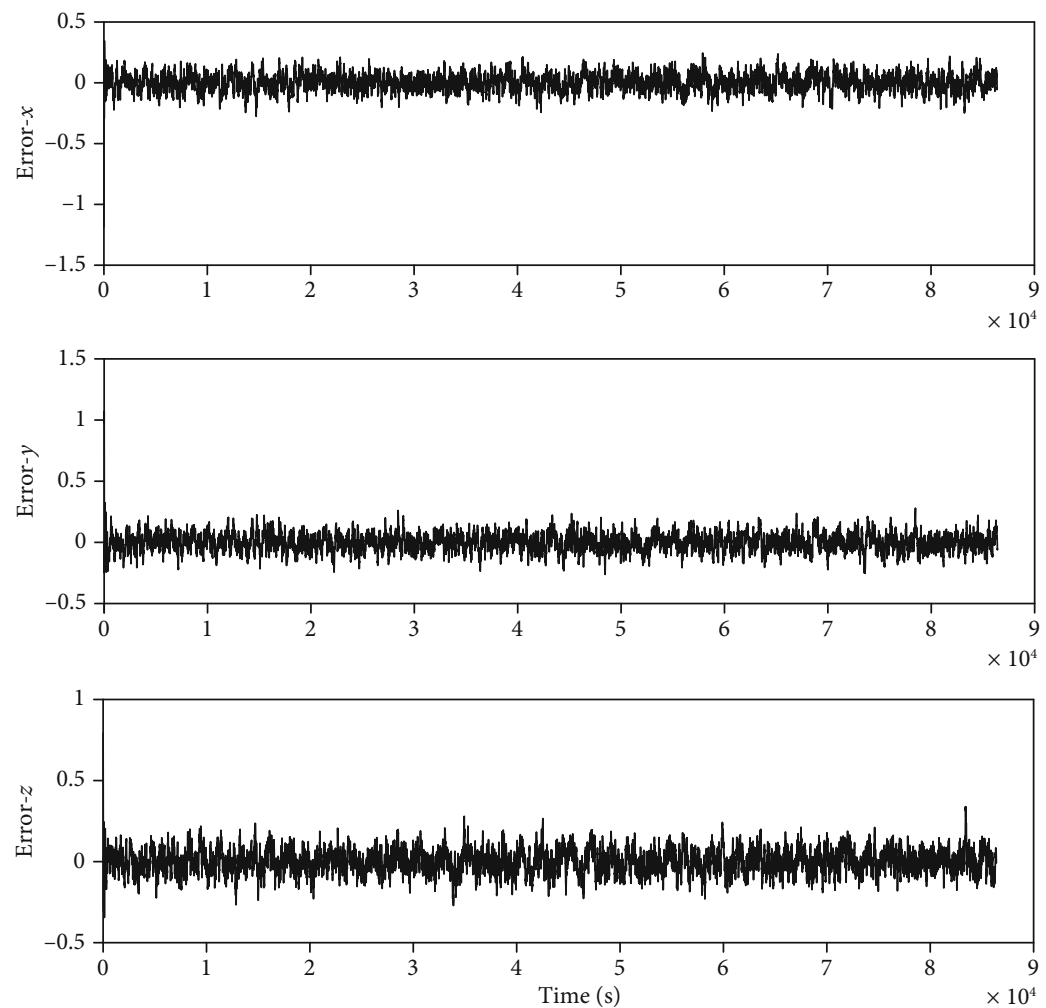

Figure 9: Position estimation error (neural network).
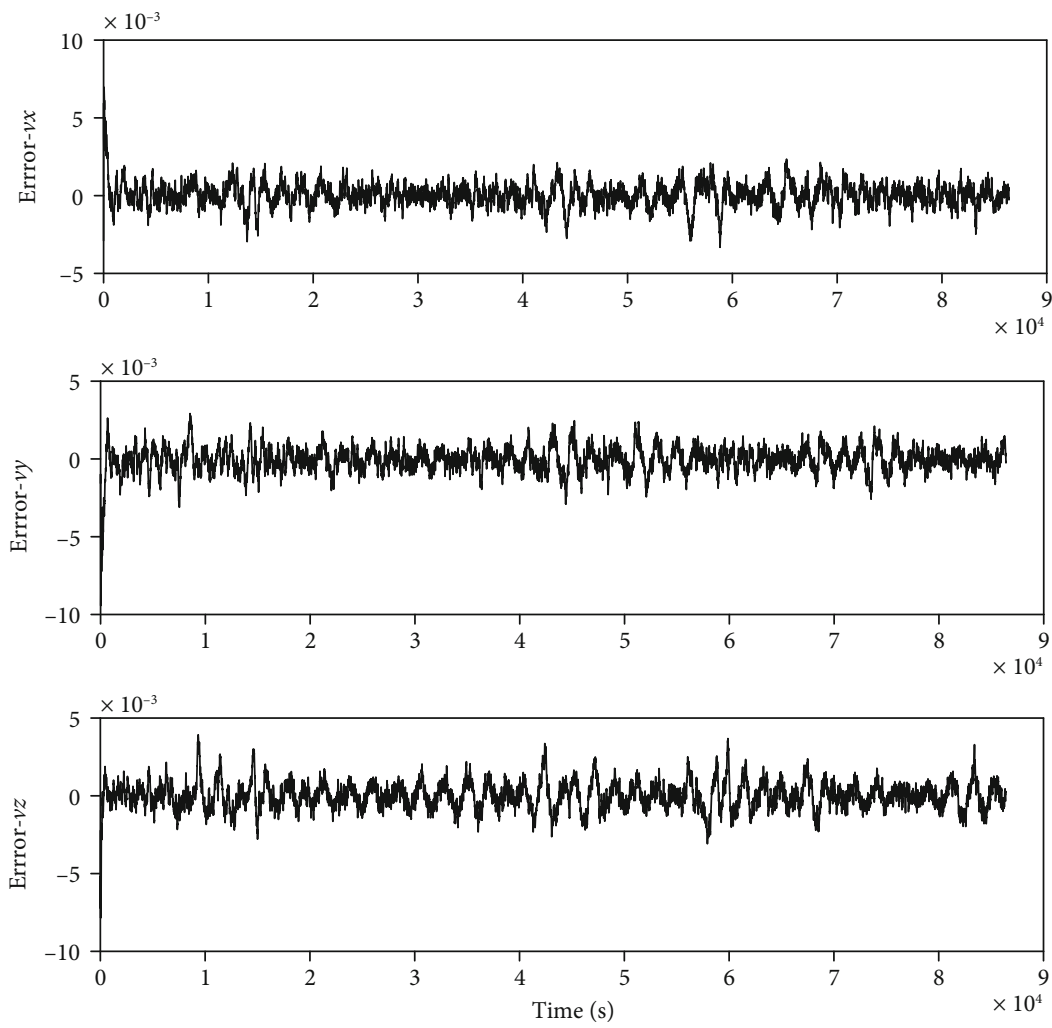

FIgURE 10: Velocity estimation error (neural network). 

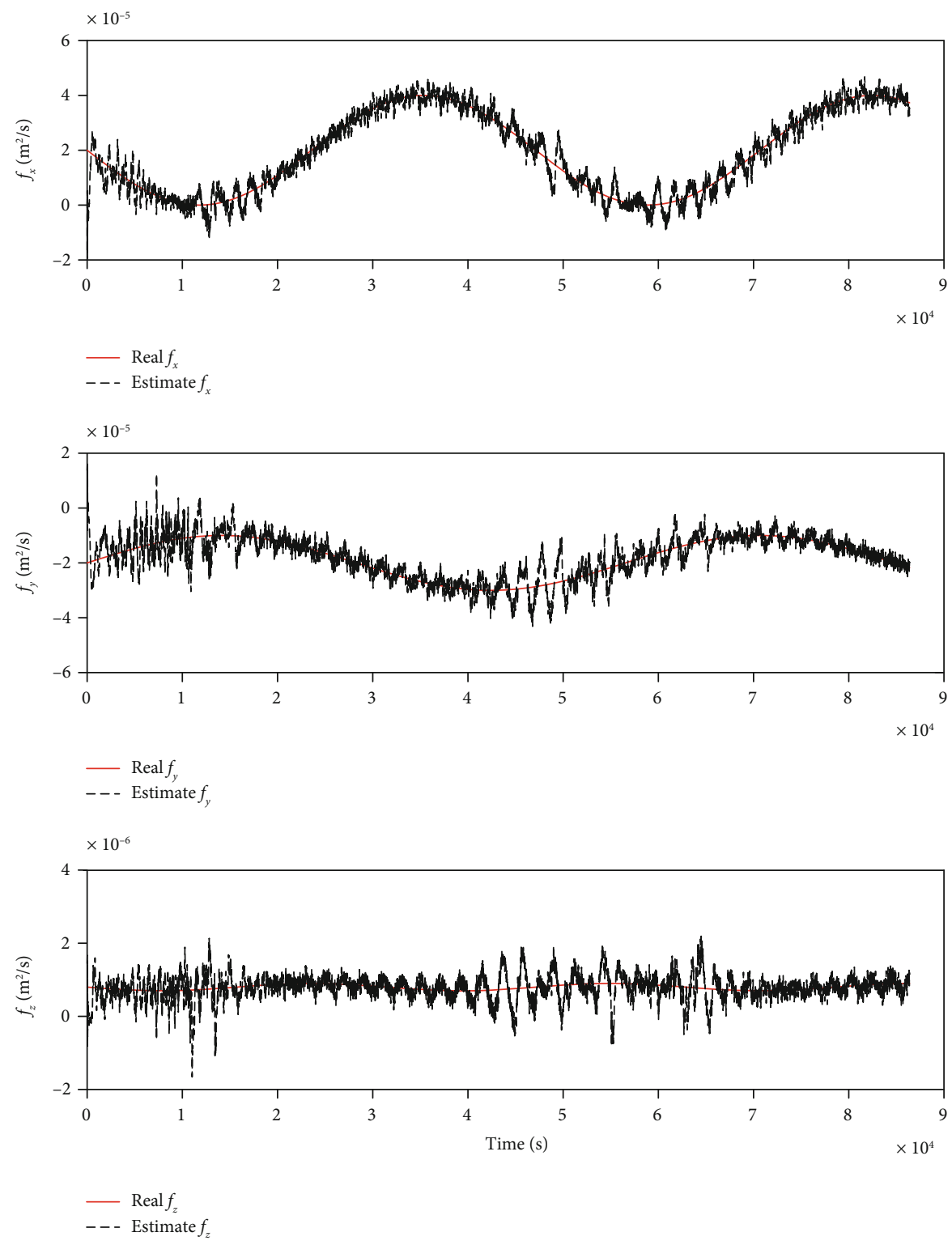

FIGURE 11: The approximation of the neural network to nonlinear terms.

The initial covariance matrix is

$$
P_{0}=\operatorname{diag}\left(\left[\begin{array}{llllll}
1^{2} & 1^{2} & 1^{2} & 0.02^{2} & 0.02^{2} & 0.02^{2}
\end{array}\right]^{T}\right) .
$$

The model error covariance matrix is selected as

$$
Q=\operatorname{diag}\left(\left[\begin{array}{llllll}
10^{-6} & 10^{-6} & 10^{-6} & 10^{-6} & 10^{-6} & 10^{-6}
\end{array}\right]^{T}\right) .
$$

The observation error is introduced in the simulation, and the assumption of the observation error is a random error with a variance of $1 \mathrm{~m}^{2}$. Therefore, the observation error covariance matrix is $R=\operatorname{diag}\left(\left[\begin{array}{lll}0.25 & 0.25 & 0.25\end{array}\right]^{T}\right)$.
The filtering results of UKF are shown in Figures 7 and 8. As we can see, the three axis position errors are under 1 meter, and the speed error is under the level of $10^{-2} \mathrm{~m} / \mathrm{s}$. It can be seen from the result graph that both the position error and the speed error have a certain degree of overall fluctuation. It can be seen that if the modeling error is large and does not meet the Gaussian distribution at all, the UKF filter is not very effective.

Experiment 3. Neural network learning rate $\eta_{c}=10^{-4}, \eta_{o}=$ $10^{-5} \cdot \kappa_{c}=\kappa_{o}=1$. And basis function $\phi_{o}(X)=\phi_{c}(X)=\left[\begin{array}{ll}1 & x / r\end{array}\right.$ $\left.y / r \quad z / r \quad x y / r^{2} \quad y z / r^{2} \quad x z / r^{2} \quad x y z / r^{3}\right], r=\sqrt{x^{2}+y^{2}+z^{2}}$. The initial state estimation and observation error settings are the same as in Experiment 2. Obtain an observation every 10 seconds. Between one observation and the next, RKF7(8) is used to solve Equation (7) to update state estimation, with 

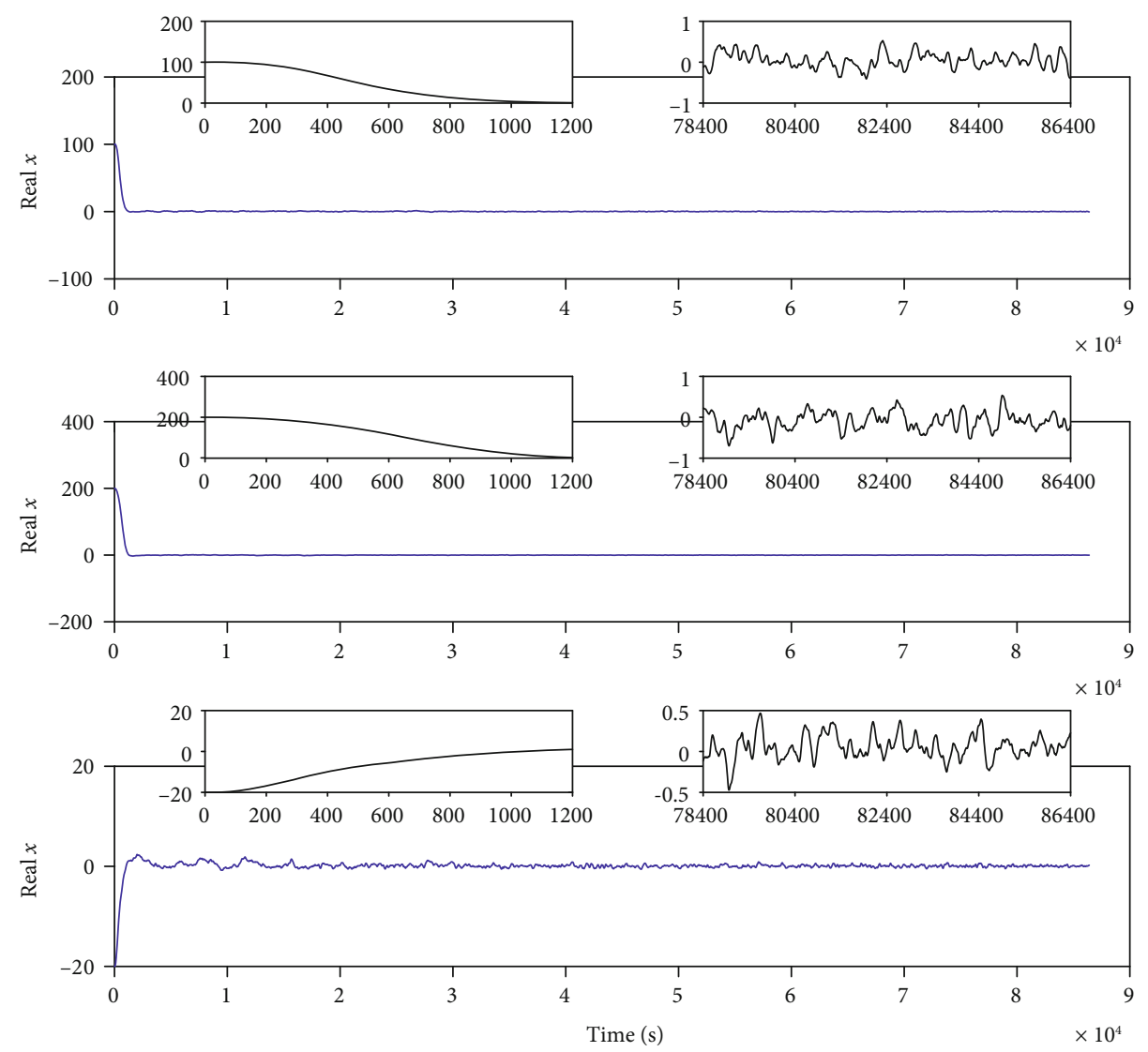

FIGURE 12: The real state change diagram.

the assumption that observation residual is not changed. Obviously, the smaller the time interval between observation, the higher the accuracy of state estimation. The observer gain matrix $K$ and the control feedback gain matrix $H$ are chosen as

$$
\begin{aligned}
K & =\left[\begin{array}{lll}
0.0152 & & \\
& 0.0152 & \\
& & 0.0152 \\
0.00019 & & \\
& 0.00019 & \\
& & 0.00019
\end{array}\right], \\
H & =\left[\begin{array}{lll}
0.0006 & 0.00047 & \\
0.00047 & 0.0006 & \\
& & 0.0006
\end{array}\right] .
\end{aligned}
$$

Figures 9 and 10 show the error between the estimated state and the real state; Figure 9 is the estimated position error in the three axes. Figure 10 is the estimation error of the speed in three axes. We can see that the state estimate of the neural network observer converges to the true value quickly. The estimation error of the position state is less than $0.5 \mathrm{~m}$, and the estimation error of the velocity is less than $0.005 \mathrm{~m} / \mathrm{s}$. Compared with the results of UKF, it can be found that the neural network method compensates the kinetic model error by estimating the perturbation, and the obtained result is relatively better. Since the perturbation equation is estimated, the prediction for the next step can be more accurate, so the smoothness of the orbit determination result is also better.

Figure 11 shows the approximation of the nonlinear term by the neural network. It can be seen from the figure that the estimation of perturbation is not a progressive approximation and eventually tends to zero, which is also consistent with the analysis in our proof of stability: the derivative of the Lyapunov function is less than 0 only when the error exceeds a certain limit. Therefore, the estimation error for perturbation is first reduced, then increased, then reduced, and then increased, but in general, the error is bounded.

Figure 12 shows the real state. It can be seen that the neural network controller can make the real state reach the target point along the designed trajectory, and the position error is within $1 \mathrm{~m}$. Figure 13 shows the control output of the neural network controller. As we can see, the output value of the neural network controller does not exceed $5 \times$ $10^{-4} \mathrm{~m} / \mathrm{s}^{2}$. This is due to the fact that this constraint has been taken into account when designing the nominal orbit. In fact, traditional controllers are often more difficult to handle when output is limited. Therefore, the neural network controller can avoid this problem by designing a reasonable nominal trajectory. 

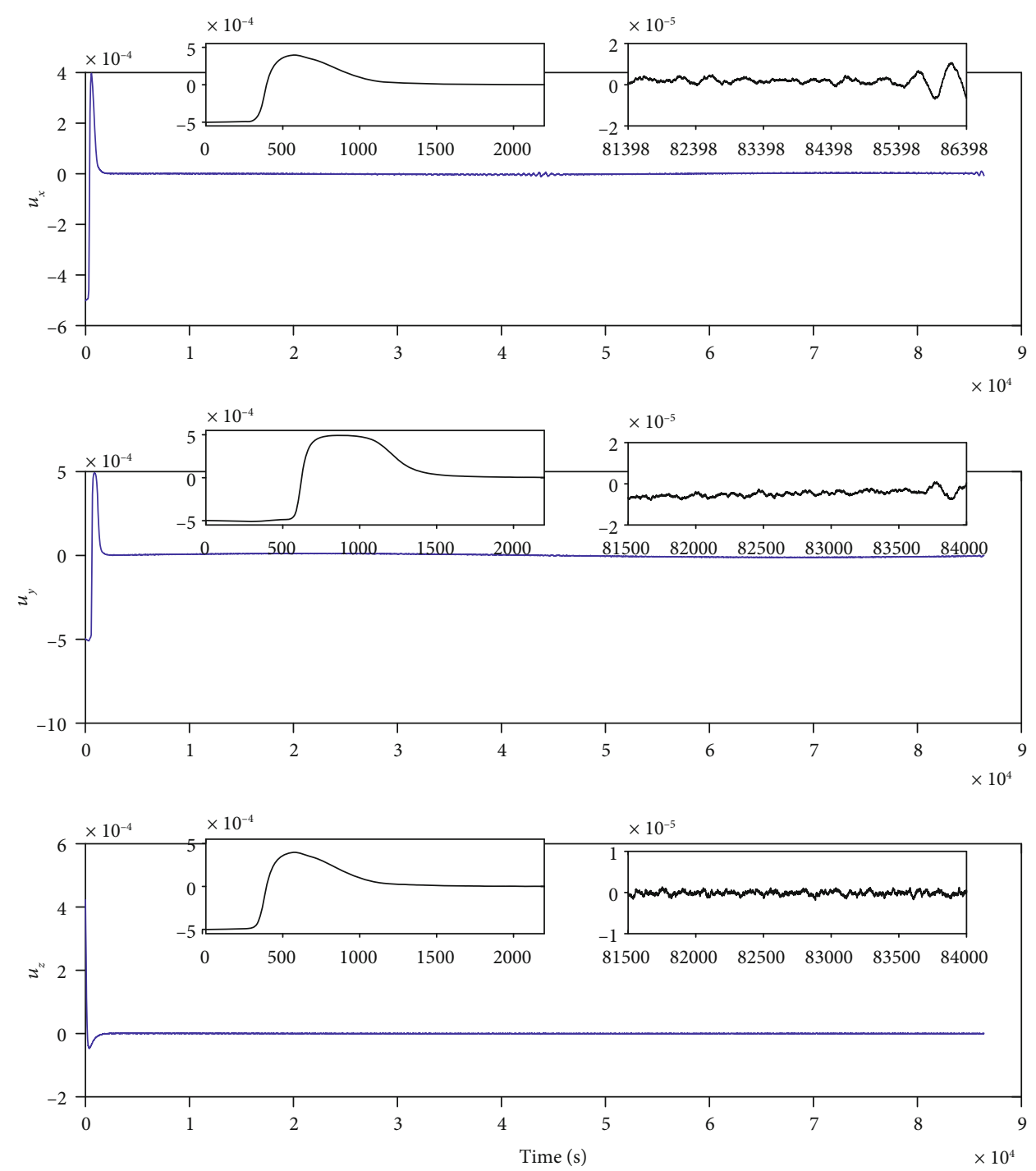

Figure 13: Controller output value.

\section{Conclusions}

Aiming at the characteristics of small maneuvering range, short duration, and difficult to accurately model for GEO satellite orbit maintenance, an integrated design method for satellite autonomous observation and control is proposed. Based on the distance between the GEO satellite and the other three satellites, the neural network state observer is designed to approximate the nonlinear term in the model and output an estimated state. Using the estimated state, the neural network controller can control the GEO satellite to the nominal position.

In the process of derivation, we assume that the small thrust controller outputs continuous and accurate thrust and that the intersatellite observations are also continuously performed and introduced into the state observer. This assumption brings convenience to our work. In order to verify the impact of these assumptions in the actual application process, we designed a numerical simulation test. In the simulation, the observations are obtained at a certain frequency, the controller output is also updated at the same frequency, and the controller execution error is added. Through the simulation results, it can be found that at the sampling frequency which is feasible under realistic conditions, the neural network observer and controller of the GEO satellite realize its functions of autonomous navigation and orbit maintenance. This proves to some extent that our observer and controller design has certain robustness and practicability. What is more, when compared to traditional UKF filter methods under the same conditions, the neural network strategy we used get better results. This is because the neural network compensates well for the error of the model. In summary, I believe that the method studied in this paper has certain practical value.

\section{Data Availability}

The data used to support the findings of this study are included within the article. We add a data availability statement in the manuscript. 


\section{Conflicts of Interest}

The authors declare that there is no conflict of interest regarding the publication of this paper.

\section{Acknowledgments}

This study was supported by the National Natural Science Foundation (No. 11705088), the Natural Science Foundation of Jiangsu Province (No. BK20160811), and the National Defense Basic Scientific Research Program of China (No. 2016110C019).

\section{References}

[1] Y. T. Gao, B. Xu, and H. H. Xiong, "A method for improving accuracy of autonomous orbit determination for navigation constellation," Journal of Astronautics, vol. 35, no. 10, pp. 1165-1175, 2014.

[2] F. Cao, X. H. Yang, Z. G. Li et al., "Orbit determination and prediction of GEO satellite of BeiDou during repositioning maneuver," Advances in Space Research, vol. 54, no. 9, pp. 1828-1837, 2014.

[3] H. Su, Precise Orbit Determination of Global Navigation Satellite System of Second Generation(GNSS-2)-Orbit Determination of IGSO, GEO and MEO Satellites, Dissertation for Doctoral Degree, University of Bundeswehr Munich, 2000.

[4] X. SONG, X. JIA, and W. JIAO, "Determining maneuver orbit of GEO using stochastic thrust model," GeomaticsandInformationScienceof WuhanUniversity, vol. 34, no. 05, pp. 573576, 2009.

[5] T. H. Xu, G. C. Xu, and X. Shen, "A maneuvered GEO satellite orbit determination using robustly adaptive Kalman filter," in ISDEA '10 Proceedings of the 2010 International Conference on Intelligent System Design and Engineering Application, Changsha, 2010.

[6] Y. B. Tian and F. Chen, The Hybrid Neural Network Technology, Science press, 2015.

[7] J. A. Vargas and E. M. Hemerly, "Neural adaptive observer for general nonlinear systems," in Proceedings of the 2000 American Control Conference. ACC (IEEE Cat. No.00CH36334), Chicago, IL, USA, USA, 2000.

[8] N. Harl, K. Rajagopal, and S. N. Balakrishnan, "Neural network based modified state observer for orbit uncertainty estimation," Journal of Guidance, Control, and Dynamics, vol. 36, no. 4, pp. 1194-1209, 2013.

[9] Y. Gao, T. Zhao, B. Jin, J. Chen, and B. Xu, “Autonomous orbit determination for Lagrangian navigation satellite based on neural network based state observer," International Journal of Aerospace Engineering, vol. 2017, Article ID 9734164, 10 pages, 2017.

[10] W. B. Gao, "Variable structure control theory basis," 1993.

[11] H. Curtis, Orbital Mechanics for Engineering Students, Butterworth-Heinemann, 3th Edition edition, 2014.

[12] W. H. Clohessy and R. S. Wiltshire, "Terminal guidance system for satellite rendezvous," Journal of the Aerospace Sciences, vol. 27, no. 9, pp. 653-658, 1960.

[13] H. H. Xiong and Y. T. Gao, "Study of satellite autonomous orbit determination in libration point quasi-periodic orbit based on elliptic restricted three-body problem model," China Sciencepaper, vol. 10, no. 4, pp. 478-487, 2015.
[14] J. K. Chen, Lagrange Satellite Maneuvering Orbit Determination Technology Based on State Observer, MSc. Dissertation, NUAA, 2017.

[15] X. Li, X. Wen, and L. S. Luo, "Fault detection of a class of nonlinear systems based on wavelet neural networks," Journal of Yancheng Institute of Technology(Natural Science Edition), vol. 29, no. 1, pp. 10-16, 2016.

[16] F. A. Shaik, S. Purwar, and B. Pratap, "Real-time implementation of Chebyshev neural network observer for twin rotor control system," Expert Systems with Applications, vol. 38, no. 10, pp. 13043-13049, 2011.

[17] Q. Liu, X. H. Ni, and X. D. Guo, "Back-stepping position controller for two-joint manipulator based on neural network state observer," Machine Tool \& Hydraulics, vol. 43, no. 3, pp. 24-28, 2015. 


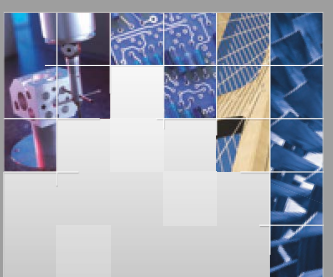

\section{Enfincering}
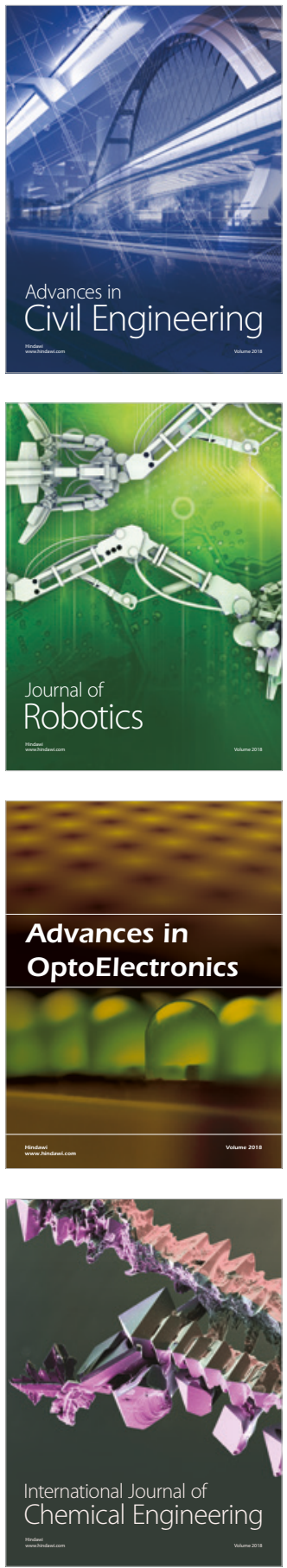

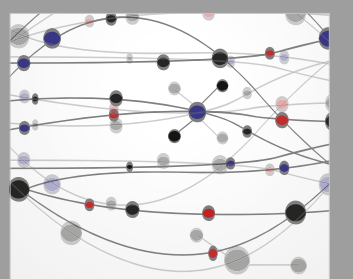

\section{Rotating \\ Machinery}

The Scientific World Journal

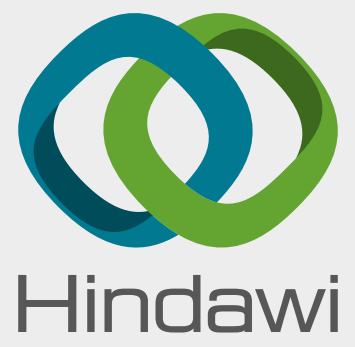

Submit your manuscripts at

www.hindawi.com
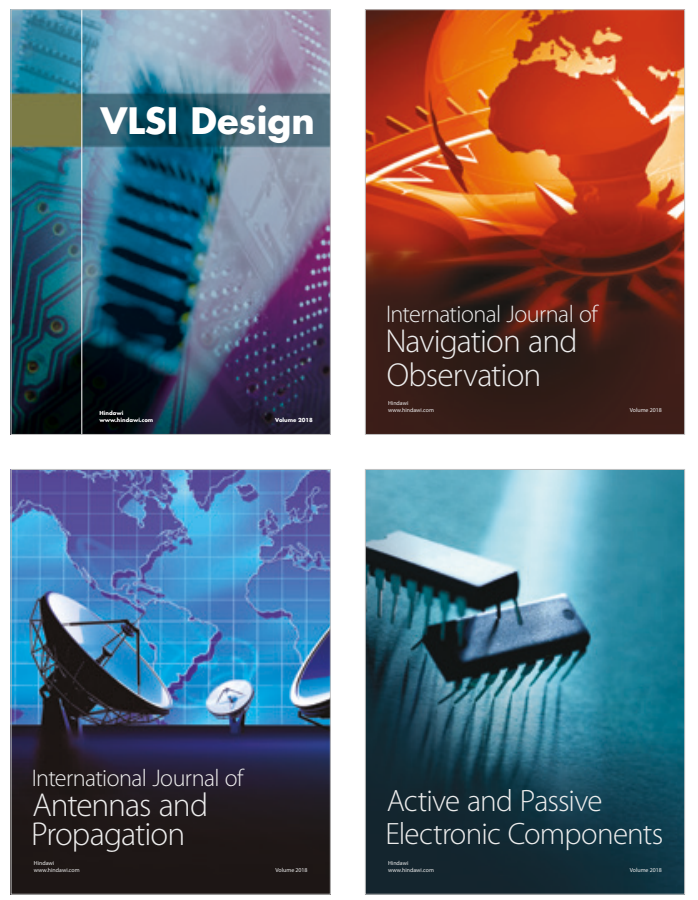
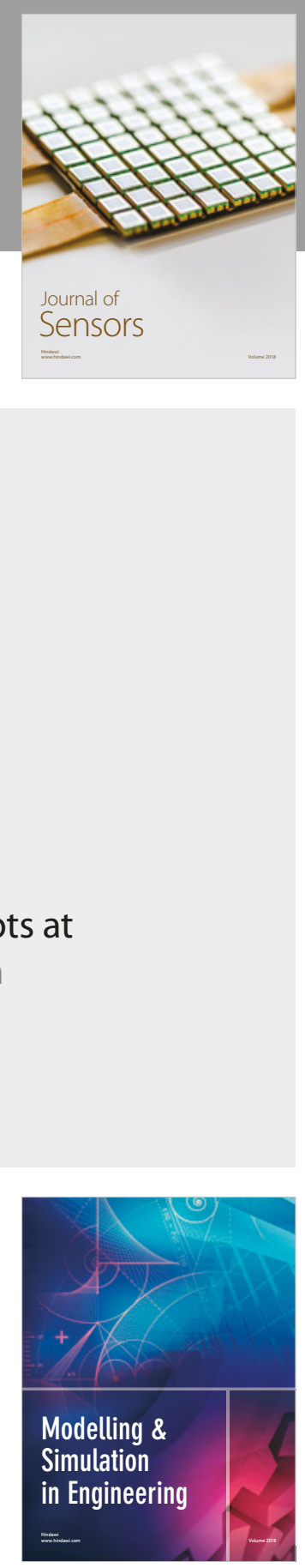

\section{Advances \\ Multimedia}
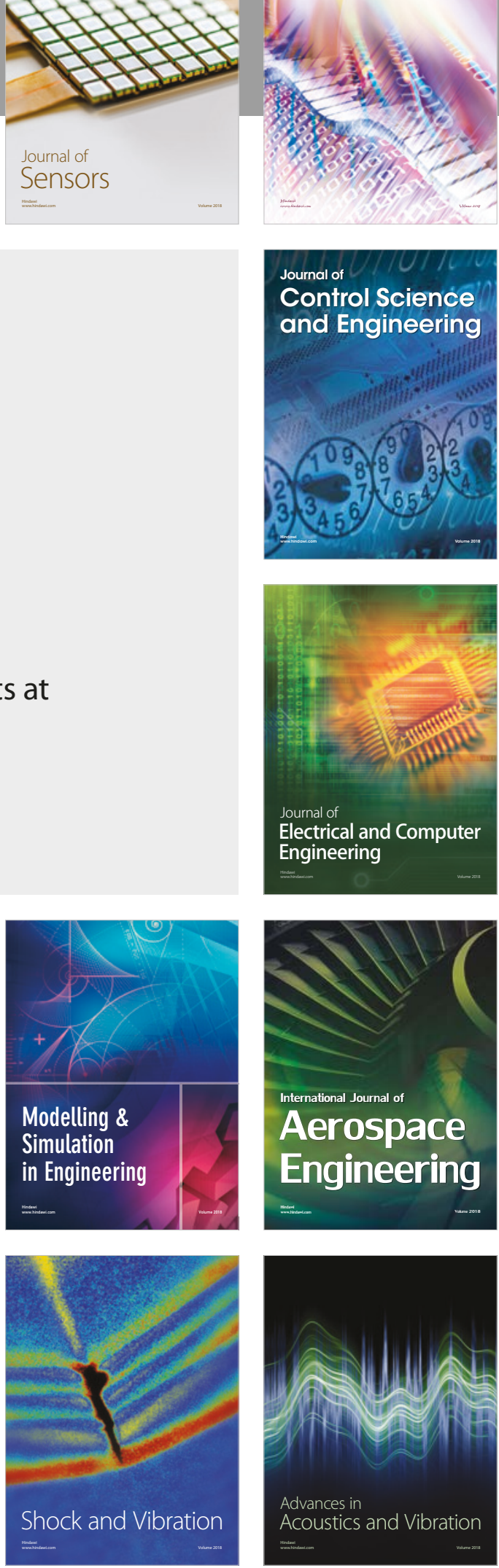